PROCEEDINGS OF THE

AMERICAN MATHEMATICAL SOCIETY

Volume 134, Number 10, October 2006, Pages 2913-2922

S 0002-9939(06)08293-1

Article electronically published on April 11, 2006

\title{
ON SOME DYADIC MODELS OF THE EULER EQUATIONS
}

\author{
FABIAN WALEFFE
}

(Communicated by Andreas Seeger)

\begin{abstract}
Katz and Pavlovic recently proposed a dyadic model of the Euler equations for which they proved finite time blow-up in the $H^{3 / 2+\epsilon}$ Sobolev norm. It is shown that their model can be reduced to a dyadic model of the inviscid Burgers equation. The inviscid Burgers equation exhibits finite time blow-up in $H^{\alpha}$, for $\alpha \geq 1 / 2$, but its dyadic restriction is even more singular, exhibiting blow-up for any $\alpha>0$. Friedlander and Pavlovic developed a closely related model for which they also prove finite time blow-up in $H^{3 / 2+\epsilon}$. Some inconsistent assumptions in the construction of their model are outlined. Finite time blow-up in the $H^{\alpha}$ norm, for any $\alpha>0$, is proven for a class of models that includes all those models. An alternative shell model of the Navier-Stokes equations is discussed.
\end{abstract}

\section{INTRODUCTION}

The Euler equations of compressible gas dynamics typically develop singularities in finite time from smooth initial conditions. The simplest example of this phenomenon is provided by the inviscid Burgers, or traffic flow, equation

$$
\frac{\partial u}{\partial t}+u \frac{\partial u}{\partial x}=0
$$

where $u=u(x, t)$ and $x$ are real, $t>0$. Let $\zeta(x, t)=\partial u / \partial x$; then from (11)

$$
\frac{\partial \zeta}{\partial t}+u \frac{\partial \zeta}{\partial x}=-\zeta^{2}
$$

By the method of characteristics, we obtain

$$
\frac{d \zeta}{d t}=-\zeta^{2}
$$

for $\zeta=\zeta(X(t), t)$, along the characteristic lines $x=X(t)$ such that $d X / d t=$ $u(X(t), t)$ with $d u / d t=0$ for $u=u(X(t), t)$. Equation (3) exhibits blow-up in finite time for negative initial conditions. Its solution is

$$
\zeta=\frac{\zeta_{0}}{1+\zeta_{0} t}
$$

which is singular at $t=-1 / \zeta_{0}>0$ if $\zeta_{0}<0$.

Received by the editors October 8, 2004 and, in revised form, April 21, 2005.

2000 Mathematics Subject Classification. Primary 35Q30, 35Q35, 76B03.

Key words and phrases. Euler equations, Burgers equation, Navier-Stokes equations, finite time blow-up.

(C)2006 American Mathematical Society Reverts to public domain 28 years from publication 
This singularity leads to finite time blow-up in the $H^{\alpha}$ Sobolev norm for $\alpha \geq 1 / 2$. The $H^{\alpha}$ norm of $u$, denoted $\|u\|_{H^{\alpha}}$, is defined in terms of the Fourier transform $\hat{u}(k, t)$ of $u(x, t)$ as

$$
\|u\|_{H^{\alpha}}^{2}=\int_{\mathbb{R}}\left(1+|k|^{2 \alpha}\right)|\hat{u}(k, t)|^{2} d k .
$$

Now, the method of characteristics provides the solution of (1) with $u(x, 0)=f(x)$ in parametric form as $u=f(\eta), x=\eta+t f(\eta)$, thus

$$
\begin{aligned}
2 \pi \hat{u}(k, t) & =\int_{\mathbb{R}} u(x, t) e^{-i k x} d x \\
& =\int_{\mathbb{R}} f(\eta) e^{-i k[\eta+t f(\eta)]}\left[1+t f^{\prime}(\eta)\right] d \eta \\
& =\frac{1}{i k} \int_{\mathbb{R}} f^{\prime}(\eta) e^{-i k[\eta+t f(\eta)]} d \eta,
\end{aligned}
$$

after integration by parts, where $f^{\prime}(\eta)=d f / d \eta$. The singularity occurs when $1+t f^{\prime}\left(\eta_{0}\right)=0$ for some $\eta_{0}$. For simplicity, assume that there is only one such $\eta_{0}$ with $f^{\prime}\left(\eta_{0}\right)<0, f^{\prime \prime}\left(\eta_{0}\right)=0$ and $f^{\prime \prime \prime}\left(\eta_{0}\right)=f_{0}^{\prime \prime \prime} \neq 0$, corresponding to the most negative initial slope. The asymptotic behavior of $\hat{u}(k, t)$ as $k \rightarrow \infty$, at the time of singularity, then follows by the method of stationary phase as

$$
\begin{aligned}
\hat{u}(k, t) & \sim \frac{1}{2 \pi i k} f^{\prime}\left(\eta_{0}\right) e^{-i k\left[\eta_{0}+t f\left(\eta_{0}\right)\right]} \int_{\mathbb{R}} e^{-i k t f_{0}^{\prime \prime \prime} s^{3} / 3 !} d s \\
& =\frac{\operatorname{Ai}(0)}{i k}\left(\frac{2}{k t f_{0}^{\prime \prime \prime}}\right)^{1 / 3} f^{\prime}\left(\eta_{0}\right) e^{-i k\left[\eta_{0}+t f\left(\eta_{0}\right)\right]},
\end{aligned}
$$

where $\operatorname{Ai}(0)=(2 \pi)^{-1} \int_{\mathbb{R}} e^{i y^{3} / 3} d y$ is the Airy function of the first kind evaluated at the origin. For example, if $f(\eta)=-\eta e^{-\eta^{2}}$, then $\hat{u}(k, 1) \sim i 3^{-1 / 3} \operatorname{Ai}(0) k^{-4 / 3}$. Since $|\hat{u}(k, t)|=O\left(k^{-4 / 3}\right)$ as $k \rightarrow \infty$, it follows that the $H^{\alpha}$ norm (5) will diverge if $\alpha \geq 5 / 6$. For non-generic initial conditions such that $f_{0}^{\prime \prime \prime}=0$, finite time blow-up can occur for smaller $\alpha$. Smooth initial conditions where $f(\eta)=-\eta$ in a finite neighborhood of the origin, for instance, lead to finite time blow-up for $\alpha \geq 1 / 2$, since $|\hat{u}(k, 1)|=O\left(k^{-1}\right)$ as $k \rightarrow \infty$ in such cases.

It is an open question whether singularities can develop in finite time for the Euler equations when the flow is incompressible. This fundamental question is related to the phenomenon of turbulence in fluid flows since classical turbulence phenomenology, as well as experimental data, suggest that the energy dissipation rate in turbulent flows tends to a strictly positive constant as the fluid viscosity tends to zero $[5]$.

Katz and Pavlovic [6] and Friedlander and Pavlovic [4 recently proposed dyadic models of the incompressible Euler equations and proved that their models exhibit finite time blow-up in the $H^{3 / 2+\epsilon}$ norm. Their proof is restricted to $0<\epsilon<2 / 3$. Here, it is shown that for a special class of initial conditions the Katz-Pavlovic model reduces to a dyadic model of the inviscid Burgers equation. The latter exhibits finite time blow-up in $H^{\alpha}$, for all $\alpha>0$. This is stronger than the unrestricted inviscid Burgers equation which shows blow-up for $\alpha \geq 1 / 2$. Some inconsistencies in the development of the Friedlander and Pavlovic model are outlined. It is shown that their model and the dyadic inviscid Burgers model belong to a class of models that exhibit finite time blow-up in $H^{\alpha}$, for all $\alpha>0$, even for weak nonlinear couplings. 


\section{Dyadic MOdel of The InViscid Burgers EQuAtion}

After the rescaling $u \rightarrow 2 u$, the inviscid Burgers equation (11) can be written in conservative form as

$$
\frac{\partial u}{\partial t}+\frac{\partial\left(u^{2}\right)}{\partial x}=0
$$

Consider periodic solutions in $-2^{-j_{0}} \pi \leq x \leq 2^{-j_{0}} \pi$, for some $j_{0} \in \mathbb{Z}$. Let $\hat{u}_{l}(t)$ be the $l$-th Fourier coefficient of

$$
u(x, t)=\sum_{l=-\infty}^{\infty} \hat{u}_{l}(t) e^{i k_{l} x}
$$

where the wavenumber $k_{l}=2^{j_{0}} l$. The Fourier transform of equation (8) reads

$$
\frac{d \hat{u}_{l}}{d t}=-i k_{l} \sum_{n=-\infty}^{\infty} \hat{u}_{n} \hat{u}_{l-n},
$$

where the time dependency of $\hat{u}_{l}=\hat{u}_{l}(t)$ has been kept implicit. Consider odd initial conditions so the solution remains odd for all times, $u(x, t)=-u(-x, t), \forall t \geq 0$. Since $u(x, t)$ is real, this anti-symmetry implies that $\hat{u}_{l}(t)$ is pure imaginary, hence, let $\hat{u}_{l}(t)=i v_{l}(t)$, where $v_{l}(t)$ is real and odd in $l$, so $v_{l}(t)=-v_{-l}(t)$, and we need only consider $l \geq 1$. Equation (10) becomes

$$
\frac{d v_{l}}{d t}=k_{l} \sum_{n=-\infty}^{\infty} v_{n} v_{l-n}=k_{l}\left(\sum_{n=1}^{l-1} v_{n} v_{l-n}-2 \sum_{n=1}^{\infty} v_{n} v_{l+n}\right) .
$$

Now, consider a model which restricts interactions to triplets of dyadic indices only. This yields a model, not an approximation, since interactions between dyadic wavenumbers generate nondyadic wavenumbers in the full equation, e.g. $n=1$, $l-n=2$ generates $l=3$. For the first sum inside the parentheses in eqn. (11), let $l=2^{m}, n=2^{p}$ and $l-n=2^{q}$. Then $2^{p}+2^{q}=2^{m}$ with $m, p, q \in \mathbb{Z}$ and $m>p, q \geq 0$. The only solution is $p=q=m-1$, with $m>0$. Likewise for the 2 nd sum in the parentheses let $l=2^{m}, n=2^{p}, l+n=2^{q}$. Then $2^{q}-2^{p}=2^{m}$ whose only solution satisfying $0 \leq m, p<q$ is $m=p=q-1$. So both sums inside the parentheses reduce to at most one term when all indices are restricted to powers of 2 . Since $l$ corresponds to wavenumber $k_{l}=2^{j_{0}} l=2^{j_{0}+m}$, a better notation for the dyadic model is to let $j=j_{0}+m$ and define $a_{j}(t)=v_{l}(t)$. Then equation (11) restricted to dyadic wavenumbers reads

$$
\begin{gathered}
\frac{d a_{j}}{d t}=2^{j}\left(a_{j-1}^{2}-2 a_{j} a_{j+1}\right), \quad j>j_{0}, \\
\frac{d a_{j_{0}}}{d t}=-2^{j_{0}+1} a_{j_{0}} a_{j_{0}+1} .
\end{gathered}
$$

\section{Reduction of the Katz-Pavlovic model}

The Katz-Pavlovic (KP) model is based, formally, on a wavelet expansion of a scalar function $u(x, t)$, with $x \in \mathbb{R}^{3}$ over the set of dyadic cubes in $\mathbb{R}^{3}$. This is the set of all cubes having sidelength $2^{-j}$ with corners on the lattice $2^{-j} \mathbb{Z}^{3}$. If $Q$ is a dyadic cube of sides $2^{-j}$, then $\tilde{Q}$ is the unique parent cube of sidelength $2^{-j+1}$ containing $Q$, and $\mathcal{C}^{1}(Q)$ is the set of all $2^{3}$ children of $Q$, each having sidelength $2^{-j-1}$. For $m \geq 1, \mathcal{C}^{m}(Q)$ denotes the set of $m$ th order grandchildren of $Q$, i.e. the set of all cubes of sidelength $2^{-j-m}$ that are contained in $Q$. 
The KP equations are not derived from the Euler equations, they are chosen to mimic the energy conserving quadratic nonlinearity of the Euler equations. The nonlinear interactions are restricted to local interactions in wavelet space and designed to push energy to smaller scales. The KP model equations for the amplitude $u_{Q}(t)$ of the wavelet localized at cube $Q$ of sidelength $2^{-j}$ is [6, eqn. (2.5)]

$$
\frac{d u_{Q}}{d t}=2^{5 j / 2} u_{\tilde{Q}}^{2}-2^{5(j+1) / 2} u_{Q} \sum_{Q^{\prime} \in \mathcal{C}^{1}(Q)} u_{Q^{\prime}} .
$$

The factors $2^{5 j / 2}$ were chosen based on the scaling properties of the Euler nonlinearity in $\mathbb{R}^{3}$ (see [6] for details).

The KP model is a tree, with each mode having 8 children. However it is a simple tree where each edge at the same level has the same weight and each cube interacts only with its unique parent and its own 8 children. Therefore if, for any cube $\tilde{Q}_{0}$ of sidelength $2^{-j_{0}}$, the initial conditions are such that all $m$ th order grandchildren of $\tilde{Q}_{0}$ have the same amplitude, i.e. if $u_{Q}\left(t_{0}\right)=u_{j}\left(t_{0}\right)$ for all cubes $Q$ of sidelength $2^{-j}$ in $\mathcal{C}^{m}\left(\tilde{Q}_{0}\right)$ (so $j=j_{0}+m$ ), for all $m>1$, then they remain so for all $t>t_{0}$. For such initial conditions, the dynamics of the $\tilde{Q}_{0}$ branch reduces to a chain model

$$
\frac{d u_{j}(t)}{d t}=2^{5 j / 2} u_{j-1}^{2}-2^{3} 2^{5(j+1) / 2} u_{j} u_{j+1},
$$

for all $j>j_{0}$. The factor $2^{3}$ in the 2 nd term on the right-hand side arises from the fact that each dyadic cube has $2^{3}$ children $Q^{\prime}$ in $\mathcal{C}^{1}(Q)$, and we now have $u_{Q^{\prime}}(t)=u_{j+1}(t)$ for all dyadic cube $Q^{\prime}$ with sidelength $2^{-j-1}$. Now if the initial conditions are also zero for all cubes with $j \leq j_{0}$ except for the cube $\tilde{Q}_{0}$, then the complete dynamics is described by the chain (14) for $j>j_{0}$ together with

$$
\frac{d u_{j_{0}}}{d t}=-2^{3} 2^{5\left(j_{0}+1\right) / 2} u_{j_{0}} u_{j_{0}+1}
$$

for the amplitude of the root cube $\tilde{Q}_{0}$.

For such initial conditions, the total energy reads

$$
E=\sum_{m=0}^{\infty} 2^{3 m} u_{j 0+m}^{2}=2^{-3 j_{0}} \sum_{j=j_{0}}^{\infty} 2^{3 j} u_{j}^{2} .
$$

The total energy is conserved by the dynamics (14), (15). Our interest is in finite energy solutions.

Define $a_{j}=2^{3 j / 2} u_{j}$. Then the energy reads $E=2^{-3 j_{0}} \sum_{j=j_{0}}^{\infty} a_{j}^{2}$, and the equations (14), (15), become

$$
\begin{gathered}
\frac{1}{8} \frac{d a_{j}}{d t}=2^{j} a_{j-1}^{2}-2^{j+1} a_{j} a_{j+1}, \quad j>j_{0}, \\
\frac{1}{8} \frac{d a_{j_{0}}}{d t}=-2^{j_{0}+1} a_{j_{0}} a_{j_{0}+1} .
\end{gathered}
$$

These equations are identical to the dyadic model for the inviscid Burgers equation (12), after a rescaling of time to eliminate the $1 / 8$ factor on the left-hand side.

\section{Friedlander-PaVlovic MOdel}

The Friedlander-Pavlovic model consists of the chain of ODEs 4, eqn. (3.10)]

$$
2 \frac{d a_{j}}{d t}=2^{5 j / 2} a_{j-1}^{2}-2^{5(j+1) / 2} a_{j} a_{j+1} .
$$


This scalar chain model is deduced from a more complex vector model. The latter vector model is constructed by drawing elements from the Katz-Pavlovic model, the Dinaburg-Sinai model [2] and the general philosophy of shell models designed to study some features of homogeneous turbulent flows (see 1] for a recent review of shell models).

The Dinaburg-Sinai model has been discussed elsewhere 8]. Briefly, it is a model of the incompressible Navier-Stokes equations in an unbounded domain, deduced by assuming that the nonlinearity is dominated by highly nonlocal interactions in Fourier space. In that model, small scales do not interact directly with each other, they evolve due to the distortion by an infinitely large scale background flow with uniform gradient. Strictly speaking, this leads to a linear model of the Navier-Stokes equations, since the large scale flow would have to be determined externally. However, Dinaburg and Sinai inconsistently define the infinitely large scale gradient as the linear superposition of the small scale gradients at the origin. This leads them to a quasilinear system of equations for the small scales (see 8 for details). The derivation of the model is physically and mathematically inconsistent, leading in particular to a lack of conservation of energy since the infinitely large scale flow contains an infinite amount of energy, but it is possible to prove that the resulting system of equations has smooth solutions for limited classes of initial conditions [2], 3].

Friedlander and Pavlovic begin with the shell model approach of considering an infinite sequence of nested shells $S_{j}$ in Fourier space with exponential spacing: $S_{j}=\left\{k \in \mathbb{R}^{3}: 2^{j-1} \leq|k|<2^{j+1}\right\}$, where $j$ is an integer. They consider a single time-dependent wavevector $k_{j}(t) \in \mathbb{R}^{3}$ with associated velocity $v_{j}(t) \in \mathbb{R}^{3}$ in each shell $S_{j}$ and define the nonlinear interactions following the Katz-Pavlovic local interactions model but with some of the structure of the Dinaburg-Sinai nonlocal interactions model. As a result, their wavevector evolution corresponds to the distortion by an infinitely large scale flow with uniform gradient, but the latter is now defined as the symmetric part of the gradient due to the next smaller scale at the origin ([6, eqn. (2.35)]). This is still physically and mathematically inconsistent from a modeling and asymptotics standpoint. It is unclear here why they choose to symmetrize their matrix $B^{T}$ which should represent the "large scale" gradient. However, since they assume that each wavevector is only distorted by the next higher wavenumber, the wavevector dynamics is relatively simple. This allows them to construct special classes of initial conditions for which the wavevector dynamics and the entire vector structure of the model disappear, except for a dynamically insignificant factor of 2 on the left-hand side of (18). The symmetrization of $B^{T}$ appears key to that scalar reduction, since if it was defined as a velocity gradient, $B^{T}=\kappa^{(j+1)}\left(v^{(j+1)}\right)^{T}$, then eqn. (3.3) in [6] would equal $\alpha^{(j+1)} / \sqrt{2}$ instead of $\alpha^{(j)} / 2$.

The Friedlander-Pavlovic scalar model (18) is structurally similar to the dyadic inviscid Burgers model (12), which we showed to be equivalent to the Katz-Pavlovic model for a special class of initial conditions. However, the interactions coefficients in (18) are $2^{5 j / 2}$ instead of $2^{j}$. This appears to be another inconsistency of the Friedlander-Pavlovic model, since they consider a single wavevector of magnitude $2^{j}$ in each shell $S_{j}$. However, their estimate for the nonlinearity, which is valid for wavelets in $\mathbb{R}^{3}$, is an overestimate for a single Fourier mode. 


\section{Finite time BlOW-UP in $H^{\alpha}$}

Here, we consider the class of chain models

$$
\begin{aligned}
\frac{d a_{j}}{d t} & =\lambda^{j} a_{j-1}^{2}-\lambda^{j+1} a_{j} a_{j+1}, \quad j>j_{0}, \\
\frac{d a_{j_{0}}}{d t} & =\quad-\lambda^{j_{0}+1} a_{j_{0}} a_{j_{0}+1},
\end{aligned}
$$

for some integer $j_{0}$, where $\lambda>1$ and the $a_{j}$ 's are real. This includes the dyadic Burgers model (12) when $\lambda=2$ and the Friedlander-Pavlovic model (18) when $\lambda=2^{5 / 2}$.

The total energy of system (19) is defined as

$$
E=\sum_{j=j_{0}}^{\infty} E_{j}<\infty, \quad E_{j}=a_{j}^{2} .
$$

It is easily verified that the total energy is preserved by the dynamics (19), thus $E(t)=E(0)$, for all $t>0$. It is also easily verified that if the initial conditions are nonnegative, $a_{j}(0) \geq 0, \forall j$, then $a_{j}(t) \geq 0, \forall j, \forall t>0$, since $d a_{j} / d t \geq 0$ if $a_{j}(t)=0$. We consider such nonnegative initial conditions hereafter.

A key characteristic of model (19) with non-negative initial data is that the energy flux is strictly toward higher wavenumbers (i.e. smaller scales). More precisely, let

$$
E_{\mathcal{B}(j)}=\sum_{l=j}^{\infty} E_{l} .
$$

Then from (19)

$$
\frac{d}{d t} E_{\mathcal{B}(j)}=2 \lambda^{j} E_{j-1} a_{j} \geq 0
$$

since $a_{j}(t) \geq 0$.

The following proof of finite time blow-up is a generalization of Lemma 5.2 and Theorem 5.3 in [4], which are themselves a rewrite of Lemma 3.2.2 and Corollary 3.2 .3 in 6 .

Lemma 1. For some $q$ with $\lambda^{-2}<q<1$, and for some $j$ sufficiently large, if

$$
E_{\mathcal{B}(j)}\left(t_{0}\right) \geq q^{j},
$$

then there is $\rho$ with $\left(\lambda^{2} q\right)^{-1 / 2}<\rho<1$, and $t$ in $\left[t_{0}, t_{0}+\rho^{j}\right]$, such that

$$
E_{\mathcal{B}(j+1)}(t) \geq q^{j+1}
$$

Proof. The proof proceeds by contradiction. Assume that (23) holds but that

$$
E_{\mathcal{B}(j+1)}(t)<q^{j+1}
$$

for all $t \in\left[t_{0}, t_{0}+\rho^{j}\right]$ and for all $\rho$ with $0<\rho<1$.

Since $E_{\mathcal{B}(j)}(t)$ is increasing with $E_{\mathcal{B}(j)}\left(t_{0}\right) \geq q^{j}$, we have

$$
E_{\mathcal{B}(j)}(t)=E_{j}(t)+E_{\mathcal{B}(j+1)}(t) \geq q^{j}, \quad \forall t \geq t_{0},
$$

and the assumption (24) then implies that

$$
E_{j}(t)>(1-q) q^{j}, \quad \forall t \in\left[t_{0}, t_{0}+\rho^{j}\right] .
$$


Now, integrating (22) written for $E_{\mathcal{B}(j+1)}(t)$, from $t_{0}$ to $t_{0}+\rho^{j}$ and using (25) yields

$$
\begin{aligned}
E_{\mathcal{B}(j+1)}\left(t_{0}+\rho^{j}\right) & =E_{\mathcal{B}(j+1)}\left(t_{0}\right)+2 \lambda^{j+1} \int_{t_{0}}^{t_{0}+\rho^{j}} E_{j} a_{j+1} d t \\
& \geq 2 \lambda^{j+1}(1-q) q^{j} \int_{t_{0}}^{t_{0}+\rho^{j}} a_{j+1} d t .
\end{aligned}
$$

The assumed upper bound (24) on $E_{\mathcal{B}(j+1)}\left(t_{0}+\rho^{j}\right)$ then gives

$$
\int_{t_{0}}^{t_{0}+\rho^{j}} a_{j+1} d t \leq \frac{q}{2(1-q) \lambda^{j+1}} .
$$

Next, consider the equation for $a_{j+1}$. Since $E_{j}=a_{j}^{2}$, it reads

$$
\frac{d a_{j+1}}{d t}=\lambda^{j+1} E_{j}-\lambda^{j+2} a_{j+1} a_{j+2} .
$$

Integrating from $t_{0}$ to $t_{0}+\rho^{j}$ and using the lower bound (25) on $E_{j}$, the upper bound (27) together with the upper bound $a_{j+2} \leq \sqrt{E_{\mathcal{B}(j+1)}}<q^{(j+1) / 2}$ which follows from (24), we obtain

$$
a_{j+1}\left(t_{0}+\rho^{j}\right) \geq q^{(j+1) / 2}\left[\lambda^{j+1} \rho^{j} q^{j / 2} \frac{1-q}{q^{1 / 2}}-\lambda \frac{q}{2(1-q)}\right] .
$$

This is in contradiction with the assumed bound $a_{j+1} \leq \sqrt{E_{\mathcal{B}(j+1)}}<q^{(j+1) / 2}$ provided

$$
\lambda \rho q^{1 / 2}>1,
$$

for some $q$ and $\rho$ with $0<q<1$ and $0<\rho<1$, and sufficiently large $j$. Since $\lambda>1$, it is always possible to pick $q$ with $\lambda^{-2}<q<1$ such that $\lambda q^{1 / 2}>1$ and then $\rho$ such that $\lambda^{-1} q^{-1 / 2}<\rho<1$, so that condition (30) can always be satisfied.

Define the $H^{\alpha}$ norm of the solution as

$$
\|a\|_{H^{\alpha}}^{2}=\sum_{j=j_{0}}^{\infty}\left(1+\left(\mu^{j}\right)^{2 \alpha}\right)\left|a_{j}\right|^{2},
$$

where $\mu^{j}$ is the wavenumber associated with amplitude $a_{j}$, with $\mu>1$. For model (19), we should pick $\mu=\lambda$, however Friedlander and Pavlovic picked $\mu=2$. A proof of finite time blow-up in $H^{\alpha}$ follows from repeated use of Lemma 1

Theorem 1. If $a_{j}(t)$ is a solution to system (19) with $a_{j}(0) \geq 0, \forall j$ and

$$
E_{\mathcal{B}(J)}(0) \geq q^{J}
$$

for some sufficiently large integer $J \geq j_{0}$ and $\lambda^{-2}<q<1$, then the $H^{\alpha}$ norm of the solution becomes unbounded in finite time, for any $\alpha>0$.

Proof. Applying Lemma 1, there is a $\rho$ with $0<\rho<1$ and a time $t_{1} \in\left[0, \rho^{J}\right]$ such that

$$
E_{\mathcal{B}(J+1)}\left(t_{1}\right) \geq q^{J+1}
$$

Iterating this argument, there is a time $t_{k} \in\left[t_{k-1}, t_{k-1}+\rho^{J+k-1}\right]$ such that

$$
E_{\mathcal{B}(J+k)}\left(t_{k}\right) \geq q^{J+k} \text {. }
$$


Then

$$
\begin{aligned}
\|a\|_{H^{\alpha}}^{2} & \geq \sum_{l=J+k}^{\infty} \mu^{2 \alpha l} a_{l}^{2}\left(t_{k}\right) \\
& \geq \mu^{2 \alpha(J+k)} \sum_{l=J+k}^{\infty} a_{l}^{2}\left(t_{k}\right) \\
& =\mu^{2 \alpha(J+k)} E_{\mathcal{B}(J+k)}\left(t_{k}\right) \geq \mu^{2 \alpha(J+k)} q^{J+k},
\end{aligned}
$$

which blows up as $k \rightarrow \infty$ provided

$$
\mu^{2 \alpha} q>1
$$

This will occur in finite time since

$$
t_{k} \leq \rho^{J}\left[1+\rho+\cdots+\rho^{k-1}\right]=\rho^{J} \frac{1-\rho^{k}}{1-\rho},
$$

which is finite as $k \rightarrow \infty$ since $0<\rho<1$.

To verify that (34) is compatible with condition (30) required by Lemma1, pick $q=\mu^{-2 \alpha+\delta}$ with $0<\delta<2 \alpha$ so that $q<1$ and (34) is satisfied, since $\mu>1$. Condition (30) requires $\lambda^{2} q=\lambda^{2} \mu^{-2 \alpha+\delta}>1$ since we need $\rho^{2}<1$. Hence, finite time blow-up for $\mu=\lambda$ requires $q=\mu^{-2 \alpha+\delta}$ with

$$
\max \{0,2 \alpha-2\}<\delta<2 \alpha,
$$

while if $\mu=2$ with $\lambda=2^{r}, r>0$, as in [4], blow-up requires

$$
\max \{0,2 \alpha-2 r\}<\delta<2 \alpha \text {. }
$$

In either case, finite time blow-up is achievable for any $\alpha>0$.

This proves that energy-conserving systems of the form (19), with $\lambda>1$, blow-up in finite time in the $H^{\alpha}$ norm for any $\alpha>0$. This applies to the Friedlander-Pavlovic model, for which $\lambda=2^{5 / 2}$, and is stronger than the finite time blow-up in $H^{3 / 2+\epsilon}$ proved in 4. Friedlander and Pavlovic concluded that if $\lambda=2^{r}$, then $r>3 / 2$ was sufficient for blow-up, but finite time blow-up in $H^{\alpha}$ occurs in fact for any $r>0$. These results also apply to the Katz-Pavlovic dyadic wavelet model which is identical, for special classes of initial conditions, to the dyadic inviscid Burgers model (12) for which $\lambda=2$. Indeed, in the notation of [6] and section 3 , with $\mathcal{D}_{j}$ denoting the set of all dyadic cubes of sidelength $2^{-j}$,

$$
\begin{aligned}
\|u\|_{H^{\alpha}}^{2} & \geq \sum_{j=j_{0}}^{\infty} 2^{2 \alpha j} \sum_{Q \in \mathcal{D}_{j}} u_{Q}^{2} \\
& \geq \sum_{j=j_{0}}^{\infty} 2^{2 \alpha j} 2^{3\left(j-j_{0}\right)} u_{j}^{2} \\
& =2^{-3 j_{0}} \sum_{j=j_{0}}^{\infty} 2^{2 \alpha j} a_{j}^{2},
\end{aligned}
$$

and this last sum can diverge in finite time by Theorem 1. Friedlander and Pavlovic choose $\lambda=2^{5 / 2}, q=2^{-3-\epsilon}$ and $\rho=2^{-\epsilon}$ with $\epsilon>0$, so $q<1$ and $\rho<1$ as required. Then condition (30) reads

$$
\lambda^{2} \rho^{2} q=2^{5-2 \epsilon-3-\epsilon}>1,
$$


which requires $0<\epsilon<2 / 3$. Katz and Pavlovic, in effect, choose $\lambda=2, q=2^{-\epsilon}$ and $\rho=2^{-\epsilon}$, since they work with $u_{j}$ instead of $a_{j}=2^{3 j / 2} u_{j}$, leading to the same restriction $0<\epsilon<2 / 3$.

\section{Concluding Remarks}

The dyadic models of the three-dimensional Euler equations that have been proposed and studied by Katz and Pavlovic 6 and Friedlander and Pavlovic 4 are in fact much more closely related to the one-dimensional Burgers equation. For the class of initial conditions discussed in section 3, the Katz-Pavlovic model is identical to a dyadic model of the inviscid Burgers equation. This is the model obtained by restricting nonlinear interactions in the one-dimensional Burgers equation to dyadic wavenumbers $k=2^{j} k_{0}, j$ integer. The Friedlander-Pavlovic model reduces to a similar system but with stronger nonlinear couplings. The dyadic model for the inviscid Burgers equation and related systems exhibit finite time blow-up in $H^{\alpha}$ for any $\alpha>0$. This is stronger than the finite time blow-up for $\alpha \geq 1 / 2$ that can occur in the unrestricted inviscid Burgers equation. This difference between the dyadic model and the full equation may suggest that nonlocal interactions limit the energy cascade to higher wavenumbers in the full equation, since the dyadic model includes only local interactions.

The idea of studying smoothness and finite time blow-up questions on simplified models of the Euler and Navier-Stokes equations has merits. Many such simplified models have been proposed and studied in the turbulence literature (see e.g. [1]). An early model, proposed by Obukhov [7, bears a strong resemblance to the dyadic model (19) discussed in this paper. The Obukhov model is

$$
\frac{1}{\lambda^{1 / 3}} \frac{d a_{j}}{d t}=\lambda^{j} a_{j-1} a_{j}-\lambda^{j+1} a_{j+1}^{2}-\nu_{j} a_{j},
$$

where $\nu_{j} \geq 0$ is a wavenumber-dependent viscosity coefficient, for instance $\nu_{j}=$ $\nu \lambda^{2 j}$, with $\nu>0$ to model Navier-Stokes. The dynamically inconsequential $\lambda^{-1 / 3}$ factor on the left-hand side has been introduced so that both models (19) and (40) have the same power-law steady states

$$
a_{j}=\lambda^{-2 / 9} \mathcal{E}^{1 / 3} \lambda^{-j / 3}
$$

in the unbounded, inviscid limit $\left(\nu=0, j_{0} \rightarrow-\infty\right)$, where

$$
\mathcal{E}=\frac{1}{2} \frac{d}{d t} E_{\mathcal{B}(j)}
$$

is the energy flux which is independent of $j$ for the power-law (41). This power law is analogous to the Kolmogorov-Obukhov $k^{-5 / 3}$ power law for the energy spectrum in three-dimensional Navier-Stokes turbulence. However, the dynamics of the two models are quite different. Model (19) is a nonlinear steepening model where energy is pushed ever more efficiently to larger wavenumbers. Model (40) is an instability cascade model. Consider, for example, initial conditions such that all modes are initially zero except $a_{l}(0)>0$, for some integer $l$. The nonlinear steepening model (19) directly starts pushing energy to $j>l$, but the energy would cascade to $j<l$ in the Obukhov model. The energy will cascade to $j=l+1$ only if $a_{l+1}(0) \neq 0$ and $\lambda^{l+1} a_{l}>\nu_{l+1}$. The cascade will continue if $a_{l+2}$ is nonzero and $\lambda^{l+2} a_{l+1}>\nu_{l+2}$, or in other words if the local Reynolds number is larger than 1 . It would be interesting to show what type of finite time blow-up is possible in the inviscid Obukhov model 
and see whether there is any connection between blow-up and the power-law (41). A connection is expected because $a_{j}>0$ is required for energy cascade to larger $j$ and $a_{j}(t)$ is guaranteed to stay positive as long as $a_{j-1} a_{j} \geq \lambda a_{j+1}^{2}$, when $\nu=0$. For power law scaling, $a_{j} \propto \lambda^{\beta j}$, this requires $\beta \leq-1 / 3$.

\section{REFERENCES}

[1] L. Biferale, "Shell models of energy cascade in Turbulence," Annu. Rev. Fluid Mech. 35, 441-468 (2003). MR 1967019 (2004b:76074)

[2] E.I. Dinaburg and Ya.G. Sinai, "A quasilinear approximation for the three-dimensional NavierStokes system," Moscow Math. J. 1, (3) 381-388 (2001). MR.1877599(2002i:76035)

[3] E.I. Dinaburg and Ya.G. Sinai, "Existence and Uniqueness of Solutions of a Quasilinear Approximation of the 3D Navier-Stokes System," Problems of Information Transmission 39, (1) 47-50 (2003). MR2101344 (2005g:35231)

[4] S. Friedlander and N. Pavlovic, "Blow-up in a three-dimensional vector model for the Euler equations," Comm. Pure Appl. Math. 57, 705-725 (2004). MR2038114(2005c:35241)

[5] U. Frisch, "Turbulence: The Legacy of A.N. Kolmogorov," Cambridge University Press, Cambridge, 1995. MR1428905 (98e:76002)

[6] N. Katz and N. Pavlovic, "Finite time blow-up for a dyadic model of the Euler equations," Trans. Amer. Math. Soc. 357 (2005), 695-708. MR2095627 (2005h:35284)

[7] A.M. Obukhov, "Some general properties of equations describing the dynamics of the atmosphere," Akad. Nauk. SSSR, Izv. Serria Fiz. Atmos. Okeana 7, No 7, 695-704 (1971).

[8] F. Waleffe, "Remarks on a quasilinear model of the Navier-Stokes equations," arXiv:math. AP/0409310, Sept. 19, 2004.

Departments of Mathematics and Engineering Physics, University of Wisconsin, MADISON, WISCONSIN 53706

E-mail address: waleffe@math.wisc.edu 